\title{
Adenocarcinoma of the Minor Duodenal Papilla: Report of a Case
}

\author{
Arichika Hoshino', Yoshiharu Nakamura ${ }^{2}$, Hideyuki Suzuki ${ }^{1}$, Satoshi Mizutani ${ }^{1}$, \\ Hideaki Ishii ${ }^{3}$, Masanori Watanabe ${ }^{1}$, Hideki Bou ${ }^{1}$, Masanori Yoshino, \\ Osamu Komine ${ }^{1}$ and Eiji Uchida ${ }^{2}$ \\ ${ }^{1}$ Institute of Gastroenterology, Nippon Medical School Musashi Kosugi Hospital \\ ${ }^{2}$ Department of Surgery, Nippon Medical School \\ ${ }^{3}$ Department of Pathology, Nippon Medical School Musashi Kosugi Hospital
}

\begin{abstract}
We report a rare case of adenocarcinoma of the minor papilla of the duodenum treated with transduodenal minor papillectomy. A 64-year-old woman was treated for an asymptomatic duodenal tumor detected on gastroduodenoscopy. Endoscopy showed a 15-mm sessile mass in the descending duodenum proximal to the major papilla. The major papilla was a villous 24-mm-diameter polypoid tumor. Histopathologic examination of the biopsy specimen showed tubular adenoma with moderate epithelial atypia. Transduodenal major and minor papillectomies were performed. The orifice of the duct of Santorini and the pancreatic duct were re-approximated to the duodenal wall to prevent acute pancreatitis caused by scarring and stenosis of the duct orifice. Histological findings were consistent with well-differentiated adenocarcinoma limited to the minor duodenal papilla, without infiltration of the duodenal wall submucosa, and confirmed complete resection. The patient had an uneventful postoperative course and has remained asymptomatic, without evidence of tumor recurrence or stenosis of the pancreatic duct orifice, for 4 years.
\end{abstract}

(J Nippon Med Sch 2013; 80: 165-170)

Key words: adenocarcinoma, minor papilla, transduodenal minor papillectomy

\section{Introduction}

Tumors of the minor duodenal papilla are rare, although adenoma ${ }^{1}$, adenomyoma ${ }^{2}$, carcinoid $^{3.4}$, and somatostatinoma ${ }^{5.6}$ have been reported. Malignancy affecting the minor papilla in the region of the accessory pancreatic duct is also rare, with only 7 reported cases $^{7-13}$. All 7 patients underwent pancreaticoduodenectomy for primary adenocarcinomas of the minor duodenal papilla. The reported frequencies of malignancy in adenomas of the papillae ranges from $26 \%$ to $65 \%^{14-16}$. Thus, adenomas of the major or minor papilla are thought to exhibit the same adenoma-carcinoma sequence as do adenomas of the colon. Although there is a consensus that papillary adenomas greater than 1.0 $\mathrm{cm}$ in size should be resected, the procedure of choice remains controversial. Traditionally, these tumors have been treated with pancreaticoduodenectomy. Early ampullary cancer is defined as a tumor limited to the mucosa of the

Correspondence to Arichika Hoshino, Institute of Gastroenterology, Nippon Medical School Musashi Kosugi Hospital, 1-396 Kosugi-cho, Nakahara-ku, Kawasaki, Kanagawa 211-8533, Japan

E-mail:kuma-3@momo.so-net.ne.jp

Journal Website (http://www.nms.ac.jp/jnms/) 


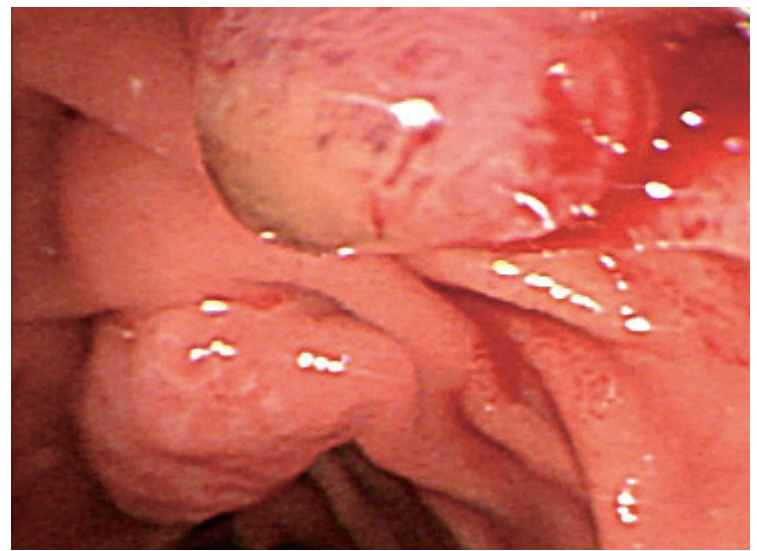

Fig. 1 Duodenoscopy with a side-view endoscope showed that the tumor had originated in the minor duodenal papilla. The major papilla was to the anal side of the minor papilla.

ampulla (depth of cancer invasion: $m$ ) or the sphincter of Oddi (depth of cancer invasion: od) regardless of the presence or absence of lymph-node metastasis ${ }^{17}$, which corresponds to pT1 of the World Health Organization classification ${ }^{18}$. We herein report a case of adenocarcinoma of the minor duodenal papilla treated with transduodenal excision of the minor papilla.

\section{Case Report}

A 64-year-old woman was admitted to our hospital for treatment of an asymptomatic duodenal tumor. The patient's general practitioner had found an irregular elevated mass in the second part of the duodenum on routine screening upper gastrointestinal endoscopy. The medical history was significant only for hypertension. The familial history included her mother who of died of gallbladder cancer and a sister with rectal cancer. The patient was in the process of quitting habitual tobacco smoking and alcohol consumption.

There were no abnormal findings on physical examination. Results of laboratory studies, including the hematological profile, renal function, pancreatic enzymes, liver enzymes, electrolytes, and tumor markers (CA19-9 and CEA), were all within normal limits. Abdominal computed tomography (CT) failed to demonstrate the primary tumor in the duodenum and did not reveal distant metastasis, lymph node

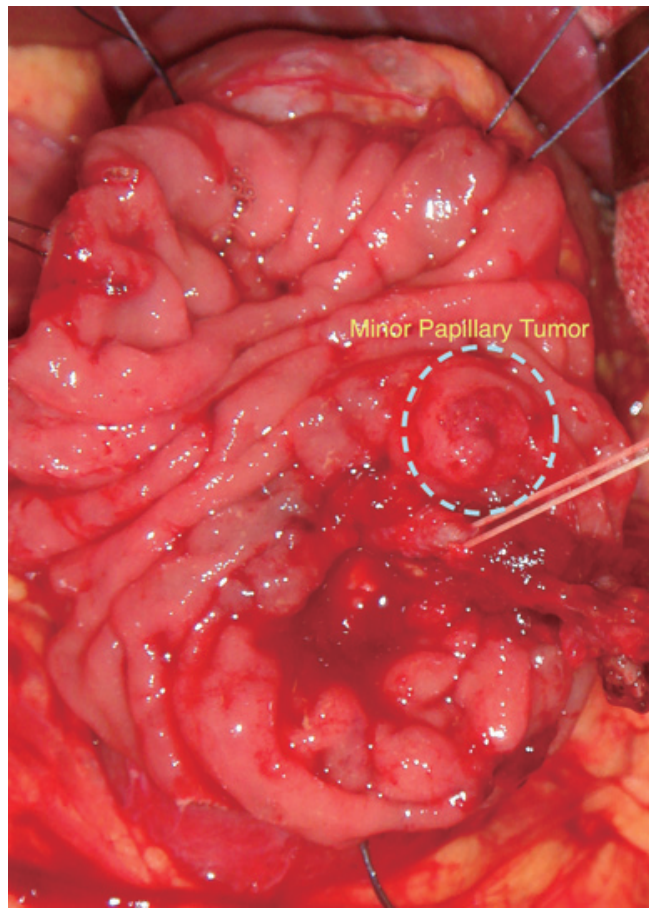

Fig. 2 The tumor in the minor papilla. Transduodenal excision of the minor papilla was performed.

metastasis, or peritoneal dissemination. Magnetic resonance cholangiopancreatography showed the pancreatic duct more clearly than did CT. Endoscopic ultrasonography failed to show any masses in the minor duodenal papilla. Screening upper gastrointestinal endoscopy revealed a $15-\mathrm{mm}$ sessile tumor in the descending duodenum proximal to the major papilla (Fig. 1). Magnifying endoscopic images showed both the major and minor papillae to be enlarged with normal surfaces. A villous polypoid tumor, approximately $24 \mathrm{~mm}$ in diameter, was observed at the major duodenal papilla. Biopsy results of these tissues were consistent with tubular adenoma with moderate epithelial atypia.

Transduodenal excision of the minor papilla including the tumor was performed. and the duct of Santorini was re-approximated to the duodenal wall with 4-0 absorbable sutures (Fig. 2). The same procedure was performed for the tumor of the major papilla. The common bile duct and the pancreatic duct were sutured and re-approximated to the duodenal wall with 4-0 absorbable sutures (Fig. 3). No prophylactic pancreatic stents were placed.

Histopathological examination of the resected 


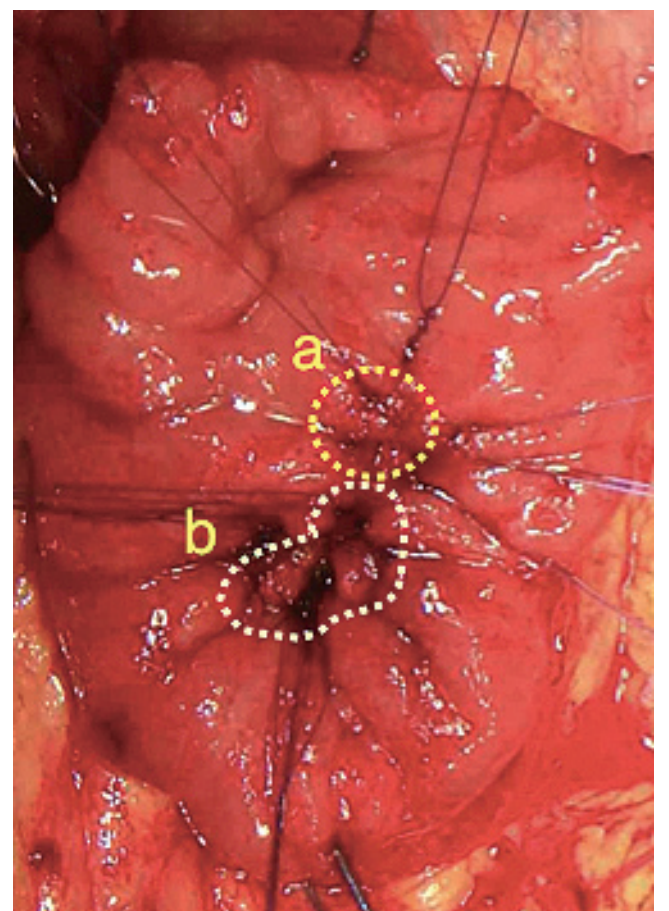

Fig. 3 (a) The duct of Santorini is re-approximated to the duodenal wall with 4-0 absorbable suture for the Santoriniplasty. (b) The common bile duct and the pancreatic duct were sutured and re-approximated to the duodenal wall with 4-0 absorbable sutures.

specimen revealed a well-differentiated adenocarcinoma within the minor duodenal papilla, without infiltration to the submucosa of the duodenal wall, and confirmed complete resection (Fig. 4). According to the World Health Organization classification $^{18}$, the histological stage of the tumor was pT1. The pathological diagnosis of the tumor of the major papilla was adenoma with moderate atypia. Additional surgical treatment was discussed with the patient and her family, but they refused. The patient had an uneventful postoperative recovery and was discharged on the 10th postoperative day.

Postoperative adjuvant therapy was not performed because of the early tumor stage. In the 4 years since the surgery, the patient has been asymptomatic without evidence of tumor recurrence or stenosis of the pancreatic and common bile duct orifice on endoscopic examination (Fig. 5).

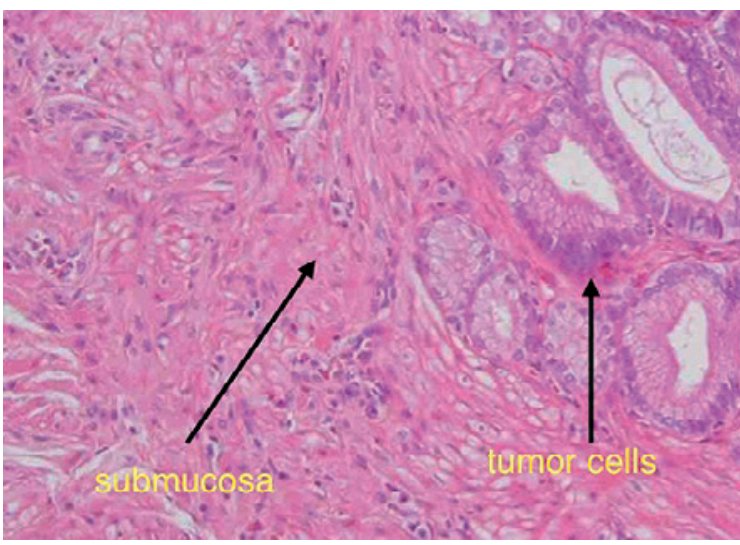

Fig. 4 Microscopic examination revealed a welldifferentiated adenocarcinoma within the minor duodenal papilla, without infiltration of the submucosa of the duodenal wall, and confirmed complete resection.

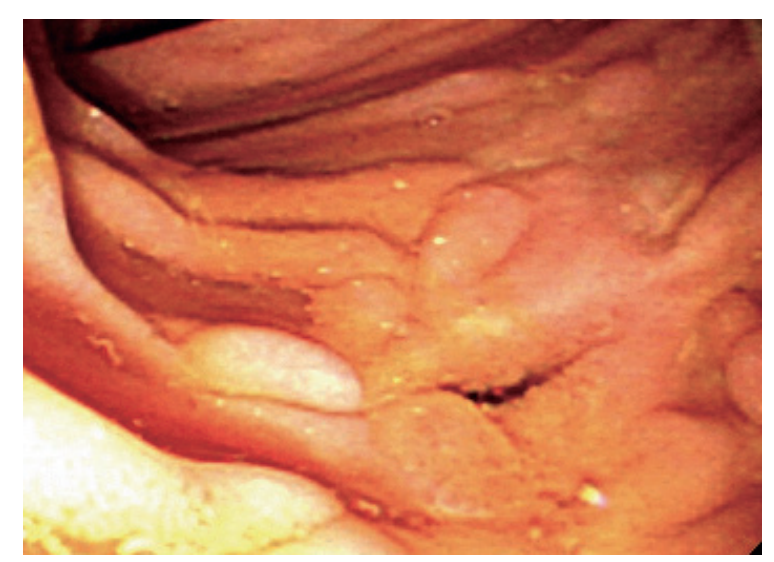

Fig. 5 Endoscopic view of the orifice of the pancreatic duct and the common bile duct 4 years after the operation. There was no evidence of tumor recurrence, and the patency of the duct of Santorini was maintained.

\section{Discussion}

Tumors in the region of the minor papilla are rare, and most of the reported cases are benign tumors, such as carcinoids ${ }^{3.4}$ and somatostatinoma ${ }^{5.6}$. In fact, the incidence of adenocarcinoma of the minor duodenal papilla, especially at an early stage, might be low ${ }^{12}$. A possible reason for the small number of reported cases is that most tumors in this area seldom produce symptoms in the early stage and are identified only at an advanced stage. The lack of characteristic symptoms makes tumors of the minor 
Table 1 Documented cases of adenocarcinoma of the minor duodenal papilla

\begin{tabular}{|c|c|c|c|c|c|c|c|c|c|}
\hline $\begin{array}{l}\text { Case } \\
\text { no. }\end{array}$ & First author & Sex & $\begin{array}{l}\text { Age } \\
\text { (years) }\end{array}$ & Chief complaint & $\begin{array}{l}\text { Size } \\
(\mathrm{mm})\end{array}$ & $\begin{array}{l}\text { Histologic } \\
\text { diagnosis }\end{array}$ & $\begin{array}{l}\text { Invasion of } \\
\text { pancreas }\end{array}$ & Treatment & $\begin{array}{c}\text { Cancer } \\
\text { of major } \\
\text { papilla }\end{array}$ \\
\hline 1 & Yamao ${ }^{7}$ & M & 82 & $\begin{array}{l}\text { Transient } \\
\text { epigastric pain }\end{array}$ & $25 \times 20$ & Mod & + & PpPD & - \\
\hline 2 & Kajiwara8) & M & 60 & $\begin{array}{l}\text { Transient } \\
\text { gastric pain }\end{array}$ & $50 \times 30$ & Well & + & SSpPD & - \\
\hline 3 & Wakatsuki ${ }^{9)}$ & M & 70 & no & $11 \times 8$ & Well & - & PpPD & - \\
\hline 4 & Parthasarathy ${ }^{10)}$ & $\mathrm{F}$ & 60 & $\begin{array}{l}\text { Fever and } \\
\text { jaundice }\end{array}$ & $15 \times 12$ & Mod & + & $\mathrm{PD}$ & + \\
\hline 5 & Matheus 11$)$ & $\mathrm{F}$ & 50 & $\begin{array}{l}\text { Abdominal pain } \\
\text { and jaundice }\end{array}$ & 10 & Mod & - & $\mathrm{PpPD}$ & + \\
\hline 6 & Okuma13) & M & 76 & no & $12 \times 10$ & Well & - & PpPD & - \\
\hline 7 & Takami12) & M & 81 & no & $20 \times 15$ & $\begin{array}{l}\text { Pap + Well } \\
+ \text { Mod }+\end{array}$ & + & $\mathrm{SSpPD}$ & - \\
\hline 8 & Present case & $\mathrm{F}$ & 64 & no & 15 & Well & - & $\mathrm{TP}$ & - \\
\hline
\end{tabular}

Mod, moderately differentiated adenocarcinoma; Well, well-differentiated adenocarcinoma; Pap, papillary adenocarcinoma; PpPD, pylorus-preserving pancreatoduodenectomy; SSpPD, subtotal stomach-preserving pancreatoduodenectomy; PD, pancreatoduodenectomy; TP, transduodenal papillectomy

duodenal papilla difficult to diagnose.

Only 7 adenocarcinomas of the minor papilla have been reported previously ${ }^{7-13}$. The present case was also asymptomatic, and the tumor, $1.5-\mathrm{cm}$ proximal to the major duodenal papilla, had been identified incidentally on screening upper gastrointestinal endoscopy. Two of the previous 7 cases (cases 4 and 5) involved synchronous carcinomas of the major and minor duodenal papillae ${ }^{10.11}$ (Table 1). Neither of these cases involved invasion of the minor papilla. The tumors of the major and minor papillae were not related. There was no pancreaticobiliary maljunction, thus they had examined with magnetic resonance cholangiopancreatography image or endoscopic retrograde cholangiopancreatography for anomaloous junction of the pancreaticobiliary duct. Of the 7 previously reported tumors of the minor duodenal papilla, 3 were asymptomatic ${ }^{9,12,13}, 2$ were associated with jaundice ${ }^{10.11}$, and 2 were associated with transient epigastralgia ${ }^{7.8}$ (Table 1). Because none of these cases had symptoms specifically associated with tumors of the minor duodenal papilla, careful observation of the papilla during routine endoscopic examinations of the upper gastrointestinal tract is essential.

In the case of the major duodenal papilla, the reported rates of malignancy involving duodenal or ampullary tumors range from $35 \%$ to $60 \%{ }^{1920}$. While the region of the ampulla is easily accessible for endoscopic biopsy, the high rate of false-negative results for carcinoma are high, from $25 \%$ to $60 \%{ }^{1920}$. Differentiating benign from malignant tumors on the basis of size or external appearance is difficult. Therefore, the tumor was completely resected in our patient because of the potentially serious problems of endoscopic biopsy and external appearance described above. We believe that adenomas of the major or the minor papilla undergo the same adenoma-carcinoma sequence as adenomas of the colon. However, a consensus has reportedly been reached that papillary adenomas greater than $1.0 \mathrm{~cm}$ in size should be resected ${ }^{21}$.

Some recently published series found endoscopic snare excision of papillary adenoma to result in favorable outcomes in terms of both safety and longterm efficacy. However, a few complications and minimal residual tumors after endoscopic resection have also been observed ${ }^{21-23}$. Some cases included acute pancreatitis due to stenosis of the pancreatic duct orifice as a late complication ${ }^{21-23}$. In the present case, the tumors were located at both the major and minor papillae. We were thus concerned that pancreatitis might develop in association with complete stenosis of the pancreatic duct orifice. Therefore, we decided to perform transduodenal excision of the minor papilla, followed by 
Santoriniplasty.

In all 7 previously reported cases, pancreaticoduodenectomy was performed to treat primary adenocarcinomas of the minor duodenal papilla $^{7-13}$ (Table 1). Preoperative examination in the present case showed the tumor to be malignant, and we thus considered pancreaticoduodenectomy including laparoscopic surgery ${ }^{24}$ to be necessary. Our patient showed no signs of malignancy preoperatively. En bloc resection was performed in case malignant cells were present in the surrounding tissues. Although Ohta and Takazaki ${ }^{25}$ have reported that the risk of nodal metastasis is low when a carcinoma of the papilla of Vater cancer does not invade beyond the sphincter of Oddi, some researchers have reported higher rates of lymphatic permeation (22\%) and nodal metastasis $(10 \%, 11 \%)$ in cases with invasion of the sphincter of Oddi ${ }^{26,27}$. In the present case, the tumor had invaded beyond the basement membrane into the sphincter of Oddi. According to the General Rules for Surgical and Pathological Studies on Cancer of the Biliary Tract by the Japanese Society of Biliary Surgery, carcinoma of the papilla of Vater confined to the mucosa and contiguous with the epithelium of the glands in the sphincter of Oddi is defined as mucosal cancer $^{17}$. There is a possibility that cancer of the minor papilla of Vater carries no risk of metastasis that invasion does not reach the mucosa. The present case has also been asymptomatic with neither macroscopic nor histological recurrence for 48 months since surgery. Consequently, the orifice of the pancreatic duct has maintained an adequate size for 4 years (Fig. 5). Our experience in this case suggests that transduodenal excision is a feasible alternative treatment for papillary tumors.

Conflict of interest statement: None of the authors has any conflicts of interest.

\section{References}

1. Akatsu T, Aiura K, Takahashi S, Kameyama K, Kitajima M, Kitagawa Y: Recurrent pancreatitis caused by ampullary carcinoma and minor papilla adenoma in familial polyposis: report of a case. Surg Today 2008; 38: 440-444.
2. Fukuda A, Yazumi S, Sawada M, et al.: Adenomyoma of the minor duodenal papilla. Gastrointest Endosc 2005; 61: 475-479.

3. Waisberg J, de Matos LL, Waisberg DR, dos Santos HV, Fernezlian SM, Capelozzi VL: Carcinoid of the minor duodenal papilla associated with pancreas divisum: case report and review of the literature. Clinics 2006; 61: 365-368.

4. Kim YG, Kim TN, Kim KO: Carcinoid tumor of the minor papilla in complete pancreas divisum presenting as recurrent abdominal pain. BMC Gastroenterology 2010; 10: 17.

5. Stömmer PE, Stolte M, Seifert E: Somatostatinoma of Vater's papilla and of the minor papilla. Cancer 1987; 60: 232-235.

6. Heidt H, Högel B, Giedl J, Gall FP, Ottenjann R: Malignant somatostatinoma of the papilla major and minor. Dtsch Med Wochenschr 1989; 114: 1740-1743.

7. Yamao K, Ohhashi K, Furukawa T, et al.: Primary carcinoma of the duodenal minor papilla. Gastrointest Endosc 1998; 48: 634-636.

8. Kajiwara K, Fujii S, Takahashi S, et al.: Adenocarcinoma of the minor duodenal papilla with intraepithelial spread to the pancreatic duct. Virchows Arch 2007; 451: 1075-1081.

9. Wakatsuki T, Irisawa A, Takagi T, et al.: Primary adenocarcinoma of the minor duodenal papilla. Yonsei Med J 2008; 49: 333-336.

10. Parthasarathy S, Pottakkat B, Yootla M, Reddy SN, Reddy KM: Synchronous adenocarcinomas of the papilla major and minor. JOP 2008; 9: 332-334.

11. Matheus AS, Jukemura J, Montagani AL, Kunitake T, Patzina RA, daCunha JE: Synchronous adenocarcinoma of the major and minor duodenal papilla. J Gastrointest Surg 2008; 12: 1301-1303.

12. Takami $\mathrm{K}$, Moriya $\mathrm{T}$, Kamiga $\mathrm{T}$, et al: Adenocarcinoma of the minor duodenal papilla: report of a case. Case Rep Gastroenterol 2011; 5: $172-178$.

13. Okuma T, Ozaki T, Abe S, et al.: Adenocarcinoma of the minor duodenal papilla: report of a case. Surg Today 2011; 41: 568-571.

14. Sobol S, Cooperman AM: Villous adenoma of the ampulla of Vater: an unusual cause of biliary colic and obstructive jaundice. Gastroenterology 1978; 75 : 107-109.

15. Seifert E, Schulte F, Stolte M: Adenoma and carcinoma of the duodenum and papilla of Vater: a clinicopathologic study. Am J Gastroenterol 1992; 87: $37-42$.

16. Perzin KH, Bridge MF: Adenoma of the small intestine: a clinicopathologic review of 51 cases and a study of their relationship to carcinoma. Cancer 1981; 48: 799-819.

17. Japanese Society of Biliary Surgery: In General Rules for Surgical and Pathological Studies on Cancer of Biliary Tract, $5^{\text {th }}$ edn. 2003; pp 68-70, Kanehara Publishing, Tokyo (in Japanese).

18. Pathology and Genetics of Tumours of the Digestive System: In World Health Organization Classification of Tumours (Hamilton SR, Aaltonen LA, eds), 2000, IARC Press, Lyon.

19. Ratter DW, Fernandez-del Castillo C, Brugge WR, Warshaw AL: Defining the criteria for local resection of ampullary neoplasms. Arch Surg 1996; 131: 361- 
371.

20. Posner S, Colletti L, Knol J, Mulholland M, Eckhauser F: Safety and long-term efficacy of transduodenal excision for tumors of the ampulla of Vater. Surgery 2000; 128: 694-701.

21. Cheng CL, Sherman S, Fogel EL, et al.: Endoscopic snare papillectomy for tumors of the duodenal papillae. Gastrointest Endosc 2004; 60: 757-764.

22. Yamao $\mathrm{T}$, Isomoto $\mathrm{H}$, Kohno $\mathrm{S}$, et al: Endoscopic snare papillectomy with biliary and pancreatic stent placement for tumors of the major duodenal papilla. Surg Endosc 2010; 24: 119-124.

23. Nakamura Y, Tajiri T, Uchida E, et al.: Adenoma of the minor papilla associated with pancreas divisum. Hepato-Gastroenterology 2007; 54: 1841-1843.

24. Nakamura Y, Matsumoto S, Yoshioka M, Shimizu T, Yamahatsu K, Uchida E: Successful laparoscopic pancreaticoduodenectomy for intraductal papillary mucinous neoplasm: a case report and a reliable technique for pancreaticojejunostomy. J Nippon Med Sch 2012; 79: 218-222.

25. Ohta T, Takazaki K: Partial resection of para amuppla portion of the duodenum for carcinoma of the ampulla of Vater. Tan-to-Sui 2003; 24: 27-32 (in Japanese).

26. Ito K, Fujita N, Noda Y, et al.: Mode of spread in early amupullary cancer in tems of establishing proper indications for endoscopic papillectomy. Dig Endosc 2004; 16: 224-228.

27. Lee SY, Jang KT, Lee KT, et al.: Endoscopic management of adenoma of the major duodenal papilla. Gastrointest Endosc 2004; 59: 225-232.

(Received, August 29, 2012)

(Accepted, December 21, 2012) 